\title{
Comparison of Feature Extraction Techniques for EEG Based Brain-Computer Interface
}

\author{
Mandeep Kaur Ghumman \\ Research Scholar, \\ IKG Punjab Technical University, \\ Jalandhar, Punjab, India \\ Email: mghumman20@yahoo.com
}

\author{
Satvir Singh \\ Associate Professor, \\ IKG Punjab Technical University \\ Jalandhar, Punjab, India \\ Email:drsatvir.in@gmail.com
}

\begin{abstract}
The analysis of electroencephalogram(EEG) signals, for implementation of brain-computer interface (BCI), has enticed a lot of interest in the research community. It can be used in a variety of applications ranging from medical rehabilitation to pleasure and entertainment. BCI is very promising research domain even in the face of a number of challenges, especially in domain of signal processing, feature extraction and that of classification techniques, as these EEG signals contain considerable amount of noise and artifacts. In this paper, various feature extraction algorithms used in BCI are investigated and compared. Common spatial pattern(CSP) is a popularly applied algorithm for extracting features from EEG signals in an implementation of BCI. Filter bank spatial pattern (FBCSP) and spectrally weighted common spatial pattern (SWCSP) are further extensions of CSP. The performance of these methods is evaluated and calculated on data set 2a of BCI competition IV, on the basis of standardized mean squared error (SMSE) - Results shows that FBCSP considerably outperforms the performance of the other methods under consideration.
\end{abstract}

Keywords-Brain-computer interface (BCI), signal processing, machine learning, electroencephalogram (EEG), common spatial pattern.

\section{Introduction}

Brain-computer interface (BCI) is an evolving technology based on computer-assisted control of devices, using signals emanating from brain activity. BCI has found applications in areas of robotics, mobility devices, environmental control, and device communication. Most of the common BCIs are based on the data fetching by use of electroencephalogram (EEG) method, owing to its noninvasive nature, as compared to other methods of data acquisition from the brain waves. The EEG based BCIs require affordable recording equipment, and can be used for facilitating real-time operation [1]. The BCI interprets and translates intentions of the subject, directly into device executable commands, without traversing the conventional neural muscular communication pathway in the human body [2]. The EEG signals are fetched from scalp of the brain,

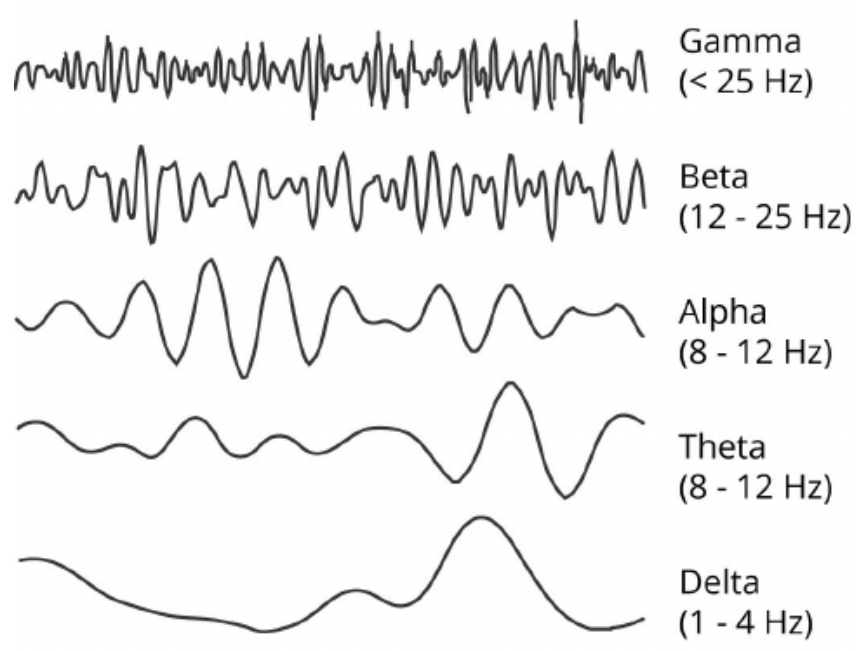

Figure 1. EEG Sub-bands [3]

which are generated due to motor imagery (MI). MI is a mere mental task of a motor act, without its actual execution by the corresponding muscle. MI is a commonly used loud mental task, wherein the subjects are cued to perform imagination of themselves executing a particular movement such as that of one of the hand or foot, without actually executing it physically. It is observed by electrical recordings from the surface of the scalp, that due to MI there is a continuous activity of the brain waves in specific locations of the brain [3]. Depending on the state of the brain, such as sleep, wakefulness or in any disease like epilepsy, the characteristics of EEG changes accordingly. The brain waves inherently lack regularity, and thus it is difficult to establish a specific pattern in the EEG signals. The EEG signals are commonly categorized into five EEG sub-bands, called as the delta band, theta band, alpha band, beta band and gamma band [4] as shown in figure 1. The activity from somatosensory and motor areas of the brain can be sensed by recording using 10-20 electrode placement system of EEG [5]. The electrodes placed in this area are labeled as C3, CZ and $\mathrm{C} 4$. The signals recorded from these labeled electrodes 


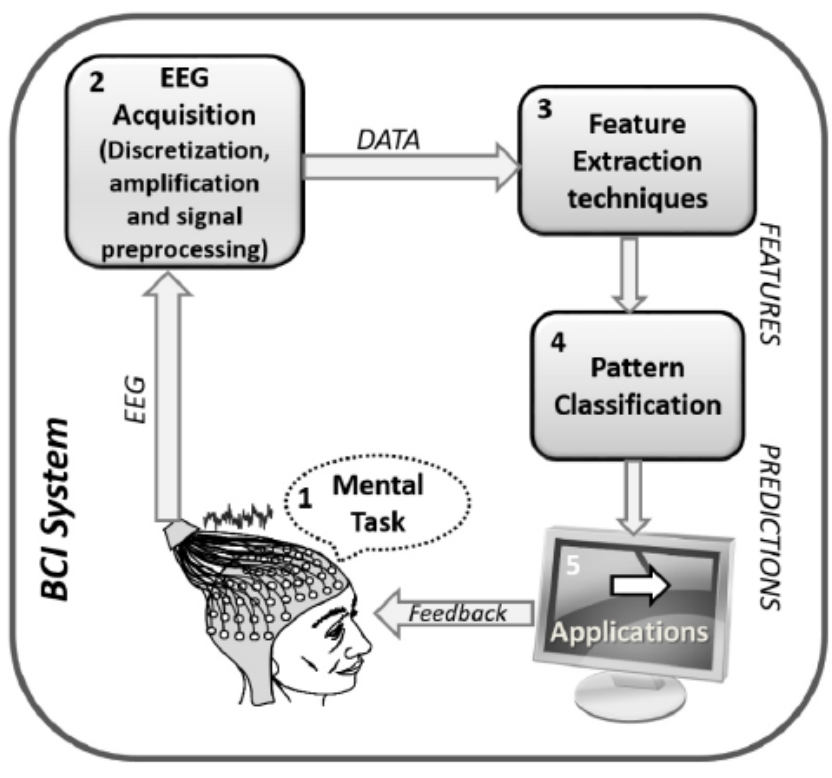

Figure 2. BCI model [8]

represent the motor activity of the subject, such as hand, foot and tongue movements etc. [6]. The EEG-based BCIs can manipulate $\mathrm{MI}$ of users, and hence can provide robust communication performance between brain and computer. There are many alternative techniques of acquiring such signals, such as that of the positron emission tomography (PET) and the magnetic resonance imaging (MRI). However EEG has emerged as preferred choice for implementation of noninvasive BCI system, due to its economic viability, higher temporal resolution and ease of use [7].

The primary application for a BCI system is to aid the disabled individuals by providing them with an alternative method of communication with some external devices. The other BCI applications include transmitting instructions in entertainment applications besides communicating with personal computers implementation of thought translation into appropriate control signals.

BCI is a multi-disciplinary research field involving neurology, rehabilitation engineering, human computer interaction (HCI), signal processing and machine learning [9]. The main objective of the BCI based applications, is to accurately translate the brain wave patterns extracted from the EEG signals into desired machine commands. The objective of many researches is to enhance this accuracy of interpretation of the harvested EEG signals [10]. Many EEG-based BCI systems were developed in recent times, in which the patterns of the EEG signals fetched in different MI tasks were analyzed, and then classified by using different algorithms at multiple stages of a BCI system as shown in figure 2. It has been observed in the research, efficiency and effectiveness of a BCI system depend on quality of algorithms employed in signal acquisition, pre-processing, feature selection and that in classification [11]. A BCI is implemented using a particular pattern recognition approach which is characterized by extraction of features from EEG signals and then using an appropriate algorithm to classify them into a mental task [12].

The EEG signals are very feeble and contain a considerable amount of noise and artifact, the process of signal processing is a difficult task.

The CSP is a commonly used algorithm for implementation of feature extraction phase of BCI.It uses the spatial filters for enhancing distractibility of two classes. CSP is an efficient algorithm used for BCI design by many researchers, especially during the BCI competitions [13]. However, the CSP has a limitation, as the frequency band which can successfully discriminate two tasks, has to be manually established by performing repeated experiments. To overcome this limitation, and for simplifying this manual frequency establishment method its enhanced variants, filter bank CSP (FBCSP) and spectrally weighted CSP (SWCSP) were proposed [14]. The techniques of collective optimization of spectral and spatial filters have has gained attention of researchers, which can be a more efficient alternative to manual selection of frequency band for each subject. This collective optimization approach falls into three broad categories. The first category contains embedded solution like common spatial spectral pattern (CSSP) and its enhanced variant called common sparse spectral spatial pattern (CSSSP). The later category perform optimization of the band pass filters the frequency domain, hence such filters are categorized as spectral filters. Different optimization techniques are iteratively exploited to lower the overheads and improve the spatial and spectral filters by use of SWCSP. Another scheme, which uses several filter bands and makes a selection of reduced set of features from predefined and narrowed bands employs FBCSP [15]. Further enhancements of CSP for carrying out multiple class classification, is an active area of research. In this research paper, we have compared the CSP, SWCSP and FBCSP on the data set 2A of the BCI competition IV [16].

\section{Data Acquisition}

In this paper, different algorithms were evaluated using the data set $2 \mathrm{~A}$ of the BCI competition IV. The BCI competition IV is in continuation to $\mathrm{BCI}$ competitions, which provides neuroscientific data, which is openly accessible to the scientific community [17]. It has data which has been live recorded from 9 human subjects while they performed some predetermined MI tasks. These EEG recording include a total of twenty five channels, from sensors which were distributed over and across sensor motor areas. Each of the 9 subjects, contributed in a calibration session and then subsequently in an evaluation session. A calibration session includes performance of four distinct MI tasks by each subject, which are categorized on basis of imagination of left hand as class 1 , right hand as class 2 , both feet as class 3 , and that of tongue as class 4 . A visual cue is shown on the computer screen as a signal to the subject, for performing a specific MI task for 4 seconds. Data set was recorded from 9 subjects, labeled as A01, A02, A03,.....A09) while they performed four-class motor MI. The nine subjects 
were seated comfortably in an arm chair, facing computer screen . The subject observed that a fixation cross appears on the black screen, marking beginning of a trial $(\mathrm{t}=0 \mathrm{~s})$. Subsequently after a gap of two seconds $(\mathrm{t}=2 \mathrm{~s})$, a further cue appears in the form of an arrow which points either to the left ,right, up or down direction. Each of the above cue corresponding to either of the four classes of left hand, right hand, tongue or foot, appears and stays on the screen for a period of $1.25 \mathrm{~s}$. This is signal to the subjects, for performing the intended MI task. There is no feedback to subjects, who are repeatedly cued to carry out the MI tasks, as per cue appearing on the screen, till the fixation cross disappears from the screen $(t=6 s)$. This procedure is punctated by a short break during which the the screen goes blank. Two such sessions were carried out for each of the subjects, wherein each of the above session constituted of 6 runs, punctuated by brief breaks in between. Each of the performed run is made up of 48 trials. Each trial is further made up of 12 trials for four identified possible classes, thus generating data from a total of 288 trials.

\section{Signal Processing}

Pre-processing and feature extraction are two important steps in EEG signal processing. Pre-processing techniques are employed to isolate unintended artifacts from the EEG signal. This leads to an improvement in the ratio of signal to noise leading to enhancement of performance of the system. Subsequently, a feature extraction process retrieves the relevant features from the input signal. The extracted features are the further fed to decision making mechanism for generating the desired classification output.

\subsection{Preprocessing}

This is the first phase signal processing, which prepares the recorded electric signal for further processing, by enhancing these so as to make them distinguishable for detection. Pre-processing techniques improve the signal and reduce the noise by rejecting unwanted artifacts. The efficiency of the preprocessing phase has a impact on the efficiency attainment of the overall BCI system. Independent component analysis (ICA) is usually applied for the removal of power line noise, as well as EOG, EMG and ECG artifacts.

\subsection{Feature Extraction}

The successful classification of MI tasks can be achieved by first extracting distinguishing and features of interest from the EEG signals. The most popular feature extraction methods is CSP, which was initially used to preprocess the two class EEG signals. It comprises of establishing linear subspaces, so that the variance value of one of the projected class is enhanced to a maximum degree. Simultaneously the variance value of the other class is reduced to a minimum. The optimal identified spatial filters are established by collective diagonalization of the two covariance matrices, which is calculated from two classes of the EEG signals. In first step, the normalized covariance matrix of each of trial fetched EEG signal E, is calculated as:

$$
R=E E^{\prime} /\left(\operatorname{trace}\left(E E^{\prime}\right)^{\prime}\right.
$$

where $\mathrm{E}$ denotes $\mathrm{n} \mathrm{X}$ t matrix, $\mathrm{n}$ denotes number of channels and $\mathrm{t}$ denotes the number of samples. The average of covariance matrices calculated from trials within a class, $\mathbf{M}_{a}$ and $\mathbf{M}_{b}$, are added to produce a comprehensive covariance matrix $\mathrm{M}_{c}=\mathrm{M}_{a}+\mathrm{M}_{b}$. The eigenvectors $\mathrm{E}_{c}$ and eigenvalues $\lambda$ of this covariance matrix result into whitening transform

$$
W=\lambda^{-1 / 2} E_{c^{\prime}}^{\prime}
$$

where $\mathrm{M}_{c}=\mathrm{E}_{c} \lambda \mathrm{E}_{c^{\prime}}$

The $\mathbf{M}_{a}$ and $\mathbf{M}_{b}$ are transformed by

$$
S_{a}=W M_{a} W^{\prime} a n d S_{b}=W M_{b} W^{\prime}
$$

The values of $S_{a}$ and $S_{b}$, are calculated by using the same Eigenvectors, in such a way that $\mathrm{S}_{a}=\mathrm{U} \psi_{a} \mathrm{U}^{\prime}$ and $\mathrm{S}_{b}=\mathrm{U} \psi_{b} \mathrm{U}$ ' wherein the $\mathrm{U}$ is calculated from the common orthonormal eigenvectors of $\mathrm{S}_{a}$ and $\mathrm{S}_{b}$ and the values of $\psi_{a}$ and $\psi_{b}$ are corresponding calculated diagonal matrices of Eigenvalues, such that thay add up to a total of 1 .

Subsequently, both classes $\mathrm{a}$ and $\mathrm{b}$ are projected onto U1 - which is the first eigenvector, leading to class a yielding the maximal value of variance and class $b$ yielding the minimal value of variance. In contrast to this, when both classes are computationally projected onto the last of the Eigenvector Um, then the class a attains the minimal value of variance whereas the class $b$ attains the maximal value of variance. In implementation, only a few of the Eigenvectors are selected, $\mathrm{U}^{*}=\mathrm{U}_{1}, \ldots \ldots . . \mathrm{U}_{m}, \mathrm{U}_{N-m+1}, \ldots \ldots \ldots \mathrm{U}_{N}$ wherein value of $\mathrm{m}$ is low $(\mathrm{m} \ll \mathrm{N})$. The finally calculated projection matrix is represented as

$$
P=U * W
$$

The signals fetched from an EEG trial, is subsequently transformed as $\mathrm{Z}=\mathrm{P} \mathrm{X}$. The attained dimension of the original signals is reduced to ' $2 \mathrm{~m}$ ' as per following equation

$$
f_{p}=\log \left(\frac{\operatorname{var}\left(Z_{p}\right)}{\sum_{p=1}^{2 m} \operatorname{var}\left(z_{p}\right)}\right), p=1, \ldots \ldots \ldots, 2 m
$$

The logarithmic transformation method is used to extract normal distributed elements in $\mathrm{f}$. The CSP, however is not applicable to multi class data with more than two classes. So for higher class problems, the FBCSP method is used.

\subsection{Filter Bank Common Spatial Pattern}

The FBCSP algorithm involves signal processing and then implementation of machine learning procedure on the EEG data. It consist of four progressive stages consisting of a filter bank consisting of band pass filters, then spatial filtering by use of CSP method, followed by a CSP based 


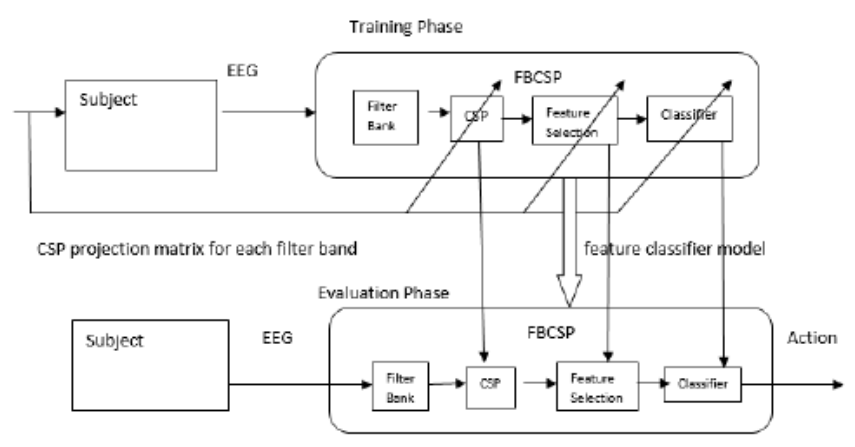

Figure 3. Filter Bank Common Spatial Pattern [18]

feature selection operation and then finally classification of the selected CSP features, as shown in figure 3. The CSP projection matrix is then calculated for each of the filter band, the discriminative CSP features, and the appropriate classifier model from the training data. It is then labeled with corresponding MI class. The parameters which are calculated from training stage are further used for classifying the MI task, in subsequent stage of evaluation phase.

3.3.1. Band-Pass Filtering. Band-pass filtering is the preliminary phase of FBCSP which makes use of filter bank to decomposes EEG signals into various frequency pass bands by use of the causal Chebyshev Type II filter. It uses nine band pass filters are used which falls in range of range of $4-8,8-12, \ldots \ldots$ and $36-40 \mathrm{~Hz}$. These ranges of band pass frequency are utilized as they cover the range of $4-40 \mathrm{~Hz}$ and achieve a stable frequency response.

3.3.2. Spatial Filtering. Spatial filtering is a second phase of FBCSP and uses the CSP algorithm as it offers higher efficiency in calculation of spatial filters, which are further used for detection of event-related desychronization/ synchronization(ERD/ERS).

The band pass and spatial filter implements the spatial filtering of EEG signals, which have previously been filtered with a particular frequency range. Each of such pair of the band pass filter and spatial filter, calculates CSP features, which belongs to a particular band pass frequency range. The stage of spatial filtering is executed by the CSP method, which linearly transforms EEG signal using

$$
Z_{b, i}=W_{b}^{T} E_{b, i}
$$

where $\mathrm{E}_{b, i} \in \mathbb{R}^{c x t}$ represents single trial EEG signal from $\mathrm{b}^{\text {th }}$ band pass filter from $\mathrm{i}^{\text {th }}$ trial; $\mathrm{Z}_{b, i} \in \mathbb{R}^{c x t}$ denotes $\mathrm{E}_{b, i}$ after implementation of spatial filtering. $\mathrm{W}_{b} \in \mathbb{R}^{c x c}$ represents the CSP projection matrix, c denotes the number of channels, $t$ denotes the number of EEG samples per channel, and $\mathrm{T}$ denotes the transpose operator.

The CSP algorithm calculates the transformation matrix $\mathrm{Wb}$, to obtain features with variances which are optimal for distinguishing two classes of the EEG signals, by resolving the eigen value decomposition problem

$$
\Sigma_{b, 1} W_{b}=\left(\Sigma_{b, 1}+\Sigma_{b, 2}\right) W_{b} D_{b}
$$

where, $\Sigma_{b, 1}$ and $\Sigma_{b, 2}$ represents the covariance matrices of EEG signals of the respective MI action which were filtered by $\mathrm{b}^{t h}$ band pass, and $\mathrm{D}_{b}$ is the diagonal matrix containing the eigen values of $\Sigma_{b, 1}$. The value of $\mathrm{W}_{b}$ is calculated in MATLAB by executing the command eig $\mathrm{S} 1$, $\mathrm{S} 1+\mathrm{S} 2$ ), where $\mathrm{S} 1$ represents $\Sigma_{b, 1}$ and $\mathrm{S} 2$ represents $\Sigma_{b, 2}$. The value of difference between variance of the two class EEG signals, which is band pass filtered, is maximized by use of $\mathrm{W}_{b}$ from equation (7). Thus, the m-pairs of CSP features from $i^{t h}$ trial of $b^{t h}$ band pass filtered EEG signals is represented by

$$
V_{b, i}=\log \frac{\operatorname{diag}\left({\overline{W_{b}}}^{T} E_{b, i} E_{b, i}^{T} \overline{W_{b}}\right)}{\operatorname{tr}\left[{\overline{W_{b}}}^{T} E_{b, i} E_{b, i}^{T} \overline{W_{b}}\right]}
$$

where $\mathrm{v}_{b, i} \in \mathbb{R}^{2 m}, \bar{W}_{b}$ denotes first $\mathrm{m}$ and last $\mathrm{m}$ columns of $\mathrm{W}_{b}$, diag(.) represents the diagonal elements of square matrix, $\operatorname{tr}[$.$] represents the summation of diagonal$ elements of square matrix. and $\mathrm{m}$ is assigned value of 2 for dataset 2a used in the experiment. The FBCSP feature vector, is then calculated for the $\mathrm{i}^{t h}$ trial in the experiment as following

$$
V_{i}=\left[V_{1, i}, V_{2, i}, \ldots \ldots, V_{9, i}\right]
$$

where $\mathrm{v}_{i} \in \mathbb{R}^{1 X(9 * 2 m)}, \mathrm{i}=1,2,3, \ldots \ldots \ldots, \mathrm{n}$; where $\mathrm{n}$ represents number of trials executed in data under consideration and $\bar{V}$ $\in \mathbb{R}^{n_{\mathrm{t}} X(9 * 2 m)}$ and $\bar{y} \in \mathbb{R}^{n_{\mathrm{t}} X 1} ; \overline{v_{i}}$ and $\overline{y_{i}}$ represents feature vector and actual class label from the $\mathrm{i}^{\text {th }}$ training session, $\mathrm{i}=1,2,3 \ldots \ldots \ldots \ldots \mathrm{n}_{t} ;$ and $\mathrm{n}_{t}$ represents the total number of trials executed in training data set under consideration.

3.3.3. Feature Selection. Measurement of brain activity, by analysis of EEG generates a huge quantity of data. The EEG signals are recorded with sampling frequency ranging from $100 \mathrm{~Hz}-1000 \mathrm{~Hz}$. These signals have to be converted into a fewer number of values characterizing some particular features of the signals like power of the EEG signals, in various frequency bands. Such features are normally further processed and aggregated into a feature vector. Feature extraction phase of the BCI, can thus be defined as a process, which converts the signals representation into an appropriate feature vector. However, the selection and extraction of relevant features, improves the performance of the system, which eases the task of further stage of classification algorithm [19]. The designer of BCI system can choose from a variety of feature selection algorithms. Their performance can then be validated, by grouping the input data into sets of training data and validation data. Feature selection is executed on the training data, by selection of distinguishing CSP features based on relation among each of the feature with corresponding MI class. The mutual information dependent best individual feature algorithm is based on the filter approach. The mutual information of each feature is calculated and sorted in descending order. The first $\mathrm{k}$ features are then selected, and are subsequently used for classification. In this research paper, we used linear discriminant analysis (LDA) classification technique. 


\subsection{Spectral Weighted Common Spatial Pattern}

SWCSP is applied for extraction of the weighted CSP feature, by using the subject-specific spectral filters. The feature extraction by implementing spectral filters and temporal spectral filters, is an important step in distinguishing different motor imaginations. In SWCSP, there is transformation of the linear time invariant band pass filters from domain of time to frequency. SWCSP performs optimization of the spatial filters by employing the CSP and spectral filters by Fisher's criterion sequentially and iteratively. Firstly, the most useful channels are selected and then extract SWCSP features at specific time interval, from signals in selected channels. Finally, all SWCSP features in different time intervals are classified by classifier to give a final result of classification, In this paper we use the LDA as a classification tool.

\subsection{Classification}

The 4th stage in implementation of BCI is a classification algorithm model which classify the selected CSP features. The objective of classification stage is automatic assignment of a class to a feature vector which is extracted in the previous stage. This class denotes the category of MI task intended by a BCI user. This classification is realized by execution of algorithms called as classifiers. Classifiers have capability to learn the identification of the class of a feature vector, by using the data from the training sets. These sets are consist of feature vectors, which are labeled with their corresponding class of MI task.

Various classification algorithms can be used for a BCI system. Linear classifiers discriminately use linear functions to demarcate different MI classes. Two of the main approaches in linear classifiers have been used for BCI design - the LDA and support vector machine (SVM). In this research paper we used the LDA classifier, which has generated better results on the dataset 2a BCI competition IV. LDA was selected due to its fastest response time among the other available classifiers [20].

3.5.1. Linear Discriminant Analysis. LDA is another popular feature classification technique [21]. LDA is used to identify linear combination of features, so as to maximize separation between two or more classes. LDA makes an assumption of normal distribution of data which has equal covariance matrices pertaining to both of the classes. It establishes a separating hyperplane, as shown in figure 5, by identifying the projection, which attempts to maximize the distance between mean of the two classes and minimizes the value of interclass variance [22]. In contrast to a 2class problem, for finding solution to a $\mathrm{N}$-class problem $(\mathrm{N}>2)$, multiple hyperplanes are required. LDA has less overheads as it has computational requirements making it more suitable for online BCI systems [23]. The LDA classifier is comparatively simple to use and offers good classification accuracy. In implementation of many MI based BCI systems, the LDA has been successfully used [19].

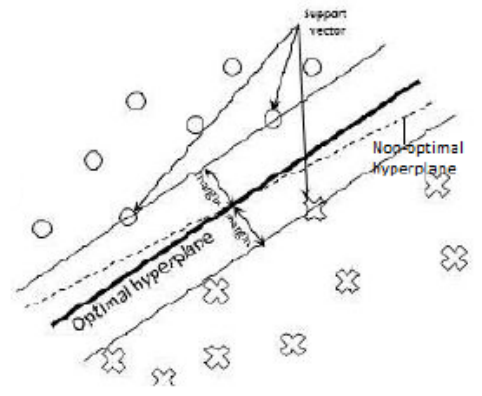

Figure 4. SVM hyperplane

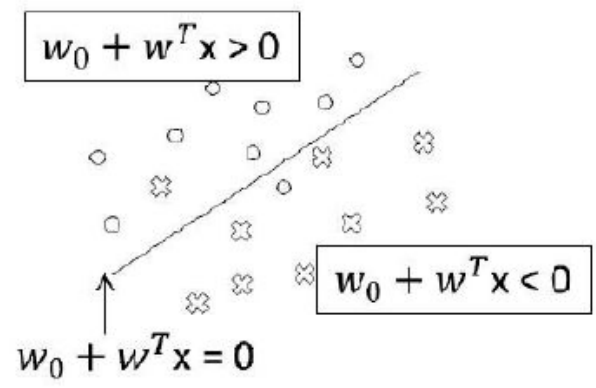

Figure 5. LDA hyperplane

\section{Simulation and Results Analysis}

The performance of various feature extraction algorithms was evaluated on data set $2 \mathrm{a}$ of BCI Competition IV. This test data composed of four classes of MI EEG data fetched from nine subjects, corresponding to MI of the left hand, right hand, feet and tongue. In above setup, two sessions were recorded from each of the subject, one for the training purpose and another for evaluation purpose. Each of the session consisted of a total of 288 trials, wherein the data was recorded by twenty two EEG channels and three monopolar electrooculogram (EOG) channels. The channel on the left mastoid acted as a reference for other channels. The performance of extraction algorithms on four class MI data fetched using the above mentioned setup was analyzed. The performance in terms of SMSE using CSP, FBCSP and SWCSP on the training data is shown in the table 1 , and graphically represented in the figure 6 .

TABLE 1. COMPARISON OF SMSE FOR CSP, FBCSP AND SWCSP

\begin{tabular}{|c|c|c|c|}
\hline & CSP & FBCSP & SWCSP \\
\hline A03T & 1.007 & 0.960 & 0.978 \\
A04T & 0.938 & 0.925 & 0.944 \\
A05T & 1.003 & 1.031 & 1.006 \\
A06T & 0.991 & 0.940 & 0.997 \\
A07T & 0.988 & 0.913 & 0.934 \\
\hline
\end{tabular}

For training of the model we selected the performance parameter of SMSE, which is presented in the table for each subject using different feature extraction algorithms. It is observed that FBCSP offers lowest SMSE as compared to the other methods. It can be observed that for every 


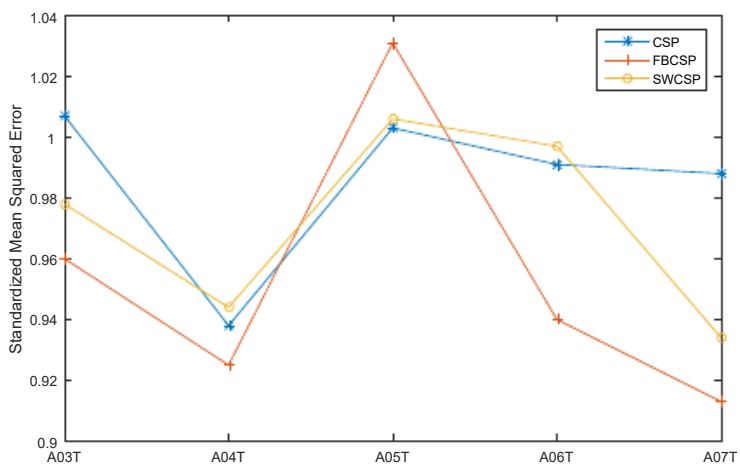

Figure 6. 5X5-fold coss-validation performance in terms of SMSE using CSP, FBCSP and SWCSP

subject, the FBCSP technique have a reduction of 0.02 to 0.03 SMSE, which is approximately one-tenth of the highest SMSE. The single trial classification performance of three approaches namely CSP, FBCSP and SWCSP, is investigated on the given training data. The classification performance is evaluated in terms of SMSE parameter using $5 * 5$ fold cross validations.

\section{Conclusion}

In this paper, we have analyzed and presented a computational methods for MI detection, and have discussed various feature extraction techniques. Feature extraction is a critical step, in the development of any EEG based BCI system. Feature extraction gives a precise and better description of the signal. In this paper, we have compared CSP, SWCSP and FBCSP feature extraction algorithms on the basis of SMSE. FBCSP shows better results than other methods. It is expected that further use of dwell and refractory periods, may further improve the performance.

\section{References}

[1] C. Park, D. Looney, N. ur Rehman, A. Ahrabian, and D. P. Mandic, "Classification of motor imagery bci using multivariate empirical mode decomposition," IEEE Transactions on neural systems and rehabilitation engineering, vol. 21, no. 1, pp. 10-22, 2013.

[2] D. Huang, K. Qian, D.-Y. Fei, W. Jia, X. Chen, and O. Bai, "Electroencephalography (eeg)-based brain-computer interface (bci): A 2$\mathrm{d}$ virtual wheelchair control based on event-related desynchronization/synchronization and state control," IEEE Transactions on Neural Systems and Rehabilitation Engineering, vol. 20, no. 3, pp. 379-388, 2012.

[3] P. JESSY, "Analysis of eeg signals for eeg-based brain-computer interface," 2009.

[4] P. Sarma, P. Tripathi, M. P. Sarma, and K. K. Sarma, "Pre-processing and feature extraction techniques for eegbci applications-a review of recent research," ADBU Journal of Engineering Technology, vol. 5, no. 1, 2016.

[5] I. Dokare and N. Kant, "Classification of eeg signal for imagined left and right hand movement for brain computer interface applications," International Journal of Application or Innovation in Engineering \& Management, vol. 2014, pp. 291-294, 2014.
[6] J. C. Lee and D. S. Tan, "Using a low-cost electroencephalograph for task classification in hci research," in Proceedings of the 19th annual ACM symposium on User interface software and technology. ACM, 2006, pp. 81-90.

[7] S. Siuly and Y. Li, "Improving the separability of motor imagery eeg signals using a cross correlation-based least square support vector machine for brain-computer interface," IEEE Transactions on Neural Systems and Rehabilitation Engineering, vol. 20, no. 4, pp. 526-538, 2012.

[8] G. Rodríguez-Bermúdez, A. Bueno-Crespo, and F. J. MartinezAlbaladejo, "Classifying bci signals from novice users with extreme learning machine," Open Physics, vol. 15, no. 1, pp. 494-500, 2017.

[9] S. G. Mason and G. E. Birch, "A general framework for braincomputer interface design," IEEE transactions on neural systems and rehabilitation engineering, vol. 11, no. 1, pp. 70-85, 2003.

[10] M. H. Alomari, A. Samaha, and K. AlKamha, "Automated classification of $1 / \mathrm{r}$ hand movement eeg signals using advanced feature extraction and machine learning," arXiv preprint arXiv:1312.2877, 2013.

[11] J. Li, L. Zhang, D. Tao, H. Sun, and Q. Zhao, "A prior neurophysiologic knowledge free tensor-based scheme for single trial eeg classification," IEEE Transactions on Neural Systems and Rehabilitation Engineering, vol. 17, no. 2, pp. 107-115, 2009.

[12] P. Herman, G. Prasad, T. M. McGinnity, and D. Coyle, "Comparative analysis of spectral approaches to feature extraction for eeg-based motor imagery classification," IEEE Transactions on Neural Systems and Rehabilitation Engineering, vol. 16, no. 4, pp. 317-326, 2008.

[13] F. Lotte and C. Guan, "Regularizing common spatial patterns to improve bci designs: unified theory and new algorithms," IEEE Transactions on biomedical Engineering, vol. 58, no. 2, pp. 355-362, 2011.

[14] G.-H. Park, Y.-R. Lee, and H.-N. Kim, "Improved filter selection method for filter bank common spatial pattern for eeg-based bci systems," International Journal of Electronics and Electrical Engineering, vol. 2, no. 2, pp. 101-105, 2014.

[15] J. Meng, L. Yao, X. Sheng, D. Zhang, and X. Zhu, "Simultaneously optimizing spatial spectral features based on mutual information for eeg classification," IEEE Transactions on Biomedical Engineering, vol. 62, no. 1, pp. 227-240, 2015.

[16] H. Zhang, C. Guan, K. K. Ang, and Z. Y. Chin, "Bci competition ivdata set i: learning discriminative patterns for self-paced eeg-based motor imagery detection," Frontiers in neuroscience, vol. 6, p. 7, 2012.

[17] M. Tangermann, K.-R. Müller, A. Aertsen, N. Birbaumer, C. Braun, C. Brunner, R. Leeb, C. Mehring, K. J. Miller, G. R. Müller-Putz et al., "Review of the bci competition iv," Frontiers in neuroscience, vol. 6, 2012.

[18] K. K. Ang, Z. Y. Chin, C. Wang, C. Guan, and H. Zhang, "Filter bank common spatial pattern algorithm on bci competition iv datasets $2 \mathrm{a}$ and 2b," Frontiers in neuroscience, vol. 6, p. 39, 2012.

[19] G. Pfurtscheller and F. L. Da Silva, "Event-related eeg/meg synchronization and desynchronization: basic principles," Clinical neurophysiology, vol. 110, no. 11, pp. 1842-1857, 1999.

[20] S. N. Resalat and V. Saba, "A study of various feature extraction methods on a motor imagery based brain computer interface system," Basic and clinical neuroscience, vol. 7, no. 1, p. 13, 2016.

[21] A. Bashashati, M. Fatourechi, R. K. Ward, and G. E. Birch, "A survey of signal processing algorithms in brain-computer interfaces based on electrical brain signals," Journal of Neural engineering, vol. 4, no. 2, p. R32, 2007.

[22] F. A. Abuhashish, M. S. Sunar, H. Kolivand, F. Mohamed, and D. B. Mohamad, "Feature extracted classifiers based on eeg signals: A survey," Life Science Journal, vol. 11, no. 4, 2014.

[23] F. Lotte, M. Congedo, A. Lécuyer, F. Lamarche, and B. Arnaldi, "A review of classification algorithms for eeg-based brain-computer interfaces," Journal of neural engineering, vol. 4, no. 2, p. R1, 2007. 